\title{
PERCEPCIONES SOBRE LA EDUCACIÓN INTERCULTURAL Y RELACIÓN MÉDICO-PACIENTE INMIGRANTE EN MÉDICOS DE CHILE
}

\author{
Ruth H. Urrutia-Arroyo ${ }^{1, a}$
}

\begin{abstract}
RESUMEN
Objetivos. Indagar en las percepciones de los médicos sobre la formación para la práctica intercultural en la relación médico-paciente (RMP) inmigrante en Chile. Materiales y métodos. Se realizó un estudio cualitativo en comunas de la región metropolitana (RM) mediante entrevistas en profundidad a médicos. Los criterios de inclusión de la muestra fueron ser médico, mantener una relación formal de trabajo en centro de salud público y haber tenido contacto con la población migrante internacional habitante de cada comuna. La muestra final fue de 13 médicos, se contempló criterios de saturación de información. Resultados. Se percibe mayor tensión relacionada con la convivencia intercultural, favorecida por la barrera cultural, idiomática y los prejuicios. La ausencia de registros sobre procedencia del paciente, contribuye a estas perspectivas. Los hallazgos dan cuenta de la ausencia de formación para la comprensión y atención de una población diversa que enfrentan a diario, así como también la necesidad de conocer el fenómeno de la inmigración en Chile y la diversidad latinoamericana actual. Conclusiones. Se ponen en discusión los nuevos desafíos a la formación médica, frente a la creciente población migrante en Chile. La emergencia de la interculturalidad en la formación médica levanta dimensiones críticas que se articulan con el enfoque intercultural y de derechos humanos en salud.
\end{abstract}

Palabras clave: Educación médica; Migración humana; Salud pública (fuente: DeCS BIREME).

\section{PERCEPTIONS REGARDING INTERCULTURAL EDUCATION IN THE DOCTOR-IMMIGRANT PATIENT RELATIONSHIP IN CHILEAN DOCTORS}

\begin{abstract}
Objective. To delve into the doctors' perceptions about training for intercultural practice in the doctor-immigrant patient (DPR) relationship in Chile. Materials and Methods. A qualitative study was carried out in communes of the metropolitan region (RM) through in-depth interviews with doctors. The inclusion criteria for the sample were: to be a doctor, to have a formal work relationship in a public health center, and to have had contact with the international migrant population living in each commune. The final sample consisted of 13 doctors; information saturation criteria were considered. Results. There is a greater tension related to intercultural coexistence, favored by the cultural, language, and prejudice barrier. The absence of records about the patients' national origin contributes to these perspectives. The findings show the lack of training geared towards understanding and caring for a diverse population on a daily basis; as well as the need to get familiar with the immigration phenomenon going on in Chile and the current Latin American diversity. Conclusions. New challenges to medical training in light of the growing migrant population in Chile are discussed here. The emergence of interculturality in medical training raises critical aspects that are articulated with healthcare intercultural and human rights approach.
\end{abstract}

Keywords: Education medical; Human migration; Public health (source: MeSH NLM).

\footnotetext{
Universidad Católica Silva Henríquez. Santiago, Chile.

Doctora en Ciencias de la Educación.

Este estudio es parte de la tesis doctoral, titulada «La relación médico-paciente inmigrante e indígena, una problemática emergente de educación intercultural desde la práctica situada de médicos formados en Universidades Estatales de la Región Metropolitana en Chile», Universidad Santiago de Chile, 2017. Recibido: 23/01/2018 Aprobado: 09/05/2018 En línea: 28/06/2018
} 


\section{INTRODUCCIÓN}

El crecimiento de la migración internacional en Chile, plantea nuevos retos a la formación médica. Para el 2015, la población extranjera con residencia en Chile, alcanzó el 2,7\% de la población total ${ }^{(1)}$, presentando un incremento de un $123 \%$ en los últimos 13 años ${ }^{(2)}$. El país se ha ido consolidando como receptor de migración heterogénea; sin embargo, el $74,9 \%$ corresponde a inmigración latinoamericana, siendo la comunidad peruana la de mayor representatividad con un $33,6 \%$. La mayor parte de los inmigrantes se encuentran en la Región Metropolitana capital del país ${ }^{(2)}$. En el marco de la salud, un $15,7 \%$ de la población inmigrante no pertenece a ningún sistema previsional de salud, mientras que en la población chilena ese porcentaje llega al $2,7 \%$, lo que constituye una barrera de acceso a la salud ${ }^{(3)}$.

La importancia del fenómeno migracional actual, no sólo tiene que ver con la densidad de la población involucrada y el impacto que ello pudiera tener en el sistema sanitario, sino porque «en este contexto nos encontramos con situaciones que dan cuenta de una construcción racializada del migrante, que utiliza rasgos fenotípicos, condición de extranjeridad y lugar de procedencia (nacionalidad) como elementos de distinción» ${ }^{(4,5)}$. El fenómeno migracional se expresa en la percepción de una diferencia cultural, representada por una «otredad» amenazante y contaminadora; también se expresa como la construcción del otro que constituye un peligro a la supuesta cultura nacional chilena, lo cual simboliza la «otredad» radical.

La migración no debiera ser algo problemático o un riesgo para la salud. Sin embargo, la evidencia internacional describe los impactos de las diferencias culturales entre el paciente y el médico que contribuyen a los malos resultados de salud. Los inmigrantes suelen tener peor salud, mayores dificultades de acceso a la atención médica, reciben cuidados de salud de menor calidad y no tienen la misma probabilidad de recibir tratamientos eficaces ${ }^{(6)}$. Uno de los aspectos de mayor impacto en el acceso a la atención de salud, es la condición migratoria de las personas ${ }^{(7)}$, lo que se agrava por factores como la discriminación y los prejuicios culturales en la toma de decisiones y recomendaciones de salud, así como por el racismo ${ }^{\left({ }^{(8)}\right.}$. Esto, en el caso de Chile, ha sido ampliamente descrito ${ }^{(9)}$.

La interculturalidad en salud, es entendida como el conjunto de acciones dirigidas a conocer e incorporar la cultura del paciente en el proceso de atención ${ }^{(10,11)}$. Más que un enfoque comunicacional, el concepto intercultural se refiere a una relación que compromete saberes culturales, educativos y de salud, de medicinas diversas en las relaciones intergrupales y entre personas ${ }^{(12)}$, combate las relaciones asimétricas y discriminatorias ${ }^{(13)}$. La interculturalidad se asocia a la política pública de salud que se orienta a superar

\section{MENSAJES CLAVE}

Motivación para realizar el estudio. La migración internacional experimenta un aumento en Chile, dando cuenta de la fluidez de un campo nuevo que transforma la práctica médica, planteando nuevos retos a la formación.

Principales hallazgos. Existen debilidades en la formación, centrada en el conocimiento de la población general, de enfoque biomédico y desconocimiento de las enfermedades de los países de origen de los inmigrantes.

Implicancias. El estudio pretende ser un aporte al debate necesario acerca de los nuevos desafíos que enfrenta la formación médica frente a la creciente población inmigrante en Chile y las exigencias del enfoque intercultural en salud.

las brechas de inequidad en las condiciones de salud de las poblaciones inmigrantes, a través de la pertinencia cultural en las acciones de salud.

En Chile, la interculturalidad es una problemática que forma parte de la política pública del sector e integra los principios orientadores para el conjunto de las áreas de la Reforma sanitaria. Según el Ministerio de Salud de Chile (2010), el enfoque Intercultural es transversal a los programas y problemas de salud. Por lo mismo, destaca la relevancia de la estrategia de formación y desarrollo de recursos humanos en materia de interculturalidad ante la necesidad de «mejorar la calidad y pertinencia cultural de las acciones de salud».

Atendiendo a que el concepto de interculturalidad actualmente es un ámbito en desarrollo y latente para la educación médica, esta investigación pretende realizar un aporte en este sentido, pero situado desde la propia experiencia (sujeto situado) de $\mathrm{RMP}^{(14)}$. Este estudio tiene como objetivo indagar en las percepciones de los médicos sobre los desafíos de formación en interculturalidad de la práctica de relación médico-paciente inmigrante en Chile. Se busca comprender los elementos que aportan en la conformación de la problemática de la interculturalidad en la formación médica y los potenciales ámbitos de acción en la política pública de salud intercultural.

\section{MATERIALES Y MÉTODOS}

\section{TIPO DE ESTUDIO}

Estudio de tipo cualitativo de carácter inductivo, diseño emergente y flexible. Se elige ese tipo de acercamiento por el carácter del tema de estudio y la problemática de interés, orientada a la comprensión del proceso formativo de los médicos desde la práctica médica caracterizada por la migración. En la perspectiva de la Epistemología del sujeto conocido de Vasilachis ${ }^{(15)}$, en la cual los sujetos no sean considerados como objetos sino como sujetos activos, con una realidad ontológica. 


\section{PROCEDIMIENTOS}

El estudio se desarrolló en cuatro comunas de la Región Metropolitana de Chile, la cual concentra la mayor densidad de población inmigrante ${ }^{(16)}$, Santiago, concentraba el 2002 el $54,4 \%$ de los extranjeros residente en la RM, seguida por Recoleta $(14,2 \%)$, Independencia $(9,6 \%)$ y Conchalí $(5 \%)$.

Para la recolección de información se utilizó la técnica de entrevistas en profundidad a informantes claves (IC). Se define la entrevista en profundidad como «reiterados encuentros cara a cara entre el investigador y los informantes, encuentros dirigidos hacia la comprensión de las perspectivas que tienen los informantes respecto de sus vidas, experiencias o situaciones, tal como las expresan con sus propias palabras» ${ }^{(17)}$, en un estilo abierto que permite obtener información intensiva, holística, contextualizada y personalizada sobre el tema de estudio.

Las entrevistas se realizaron en los ambientes naturales y cotidianos de los participantes asegurando la privacidad y confidencialidad de la conversación y donde fuera posible hablar sin interrupciones y el informante se sintiera relajado. Se llevaron a cabo en sala de atención que dispone el médico en atención primaria y sala de uso médico para las áreas de urgencia y hospitalarias en horarios en que no realizaban consulta, lo que evitó interrupciones asegurando la intimidad de la conversación. Las entrevistas abiertas tuvieron una duración de 60 a 90 minutos, se basó en un guión general y flexible en su manejo (Tabla 1). Se efectuó una prueba piloto a dos médicos con similares características de la muestra, con el objeto de identificar posibles sesgos, imposición de conceptos y la capacidad de profundizar en la conversación a partir del propio relato de los entrevistados.

Las entrevistas fueron realizadas por el investigador a cargo del proyecto original, fueron grabadas, con el consentimiento explícito del entrevistado y posteriormente se transcribieron textualmente. La recolección de datos se realizó en el 2015.

\section{PARTICIPANTES}

La selección de los sujetos fue de manera deliberada e intencionada. Se realizaron un total de 13 entrevistas individuales en profundidad. La Tabla 2 describe a los participantes del estudio. Los criterios de inclusión fueron ser médico, mantener una relación formal de trabajo en centro de salud público y haber tenido contacto con la población migrante internacional habitante de cada comuna. Se consideraron sólo egresados de las universidades tradicionales y públicas de la RM que imparten la carrera de medicina. La muestra final fue de 13 participantes: nueve médicos de atención primaria y cuatro médicos de hospital de carácter docente asistencial en convenio con universidad pública para la formación clínica de sus estudiantes, por lo cual los entrevistados desarrollaban labores de docencia clínica en sus respectivos servicios clínicos.

La variedad de tipos de centros de salud obedece a la búsqueda de profundizar en distintas perspectivas en relación al fenómeno de estudio. Para el reclutamiento de los participantes se contactaron directores, y a través de redes

Tabla 1. Guión de las entrevistas en profundidad en médicos que atienden inmigrantes en establecimientos públicos de salud de Chile

\begin{tabular}{|c|c|c|}
\hline Objetivo & Tópico & Pregunta \\
\hline \multicolumn{3}{|c|}{ Percepción acerca de la relación médico paciente inmigrante } \\
\hline $\begin{array}{l}\text { Develar la forma en que los } \\
\text { discursos de un grupo de médicos } \\
\text { formados en universidades } \\
\text { estatales, reconoce al inmigrante en } \\
\text { la experiencia de relación médico- } \\
\text { paciente en salud pública }\end{array}$ & $\begin{array}{l}\text { El inmigrante en la relación } \\
\text { médico paciente. } \\
\text { Percepción de diversidad en la } \\
\text { relación médico paciente. } \\
\text { Reconocimiento de la diversidad } \\
\text { a través de registro formal sobre } \\
\text { procedencia paciente inmigrante. }\end{array}$ & $\begin{array}{l}\text { ¿Cuál es su experiencia de atención de } \\
\text { paciente inmigrante? } \\
\text { ¿Cómo reconoce usted a este paciente? } \\
\text { ¿Existe un registro de la procedencia/ } \\
\text { origen del paciente inmigrante en la } \\
\text { RMP? }\end{array}$ \\
\hline \multicolumn{3}{|c|}{ Desafíos de la educación intercultural a la formación médica en la relación médico-paciente inmigrante } \\
\hline $\begin{array}{l}\text { Caracterizar las percepciones que } \\
\text { tienen los médicos en torno a su } \\
\text { preparación profesional para la } \\
\text { práctica de relación médica con } \\
\text { personas inmigrantes }\end{array}$ & $\begin{array}{l}\text { La educación intercultural, en } \\
\text { torno a la preparación profesional } \\
\text { para la práctica médica con } \\
\text { persona inmigrante. } \\
\text { Las necesidades de formación } \\
\text { médica para la práctica de RMP } \\
\text { inmigrante. }\end{array}$ & $\begin{array}{l}\text { ¿Cómo le prepararon para abordar esta } \\
\text { experiencia profesional? } \\
\text { En su opinión, ¿Qué necesidades de } \\
\text { formación percibe necesarias para } \\
\text { abordar la relación médico-paciente } \\
\text { inmigrante? }\end{array}$ \\
\hline
\end{tabular}


Tabla 2. Características de muestra de médicos de establecimientos de salud pública que atienden pacientes inmigrantes

\begin{tabular}{lll}
\hline Característica & $\mathbf{n}$ & \% \\
\hline Sexo & 6 & 54 \\
$\quad$ Hombre & 7 & 46 \\
$\quad$ Mujeres & & \\
$\begin{array}{l}\text { Periodo de años de obtención de } \\
\text { titulación }\end{array}$ & 2 & 15 \\
$\quad \begin{array}{l}\text { 1962-1999 } \\
2000-2010\end{array}$ & 11 & 85 \\
$\begin{array}{l}\text { Nivel de atención } \\
\quad \text { Atención primaria }\end{array}$ & 9 & 69 \\
$\quad$ Hospitalaria & 4 & 30 \\
\hline $\begin{array}{l}\text { Servicio de Salud } \\
\quad \text { Metropolitano Centro (Santiago) }\end{array}$ & 6 & 46 \\
$\quad \begin{array}{l}\text { Metropolitano Norte (Recoleta, } \\
\text { Independencia, Conchalí) }\end{array}$ & 7 & 54 \\
\hline
\end{tabular}

de conocidos de los participantes, en centros de salud que presentaban población migrante internacional inscrita formal o informalmente.

\section{ANÁLISIS}

El análisis de contenidos se realizó siguiendo las siguientes etapas: 1. Realizada la transcripción literal se obtuvo el cuerpo de contenidos. Antes de iniciar la codificación descriptiva e interpretativa se definieron las unidades de análisis que corresponden a los trozos de contenido sobre los cuales se comenzó a elaborar los análisis. Es decir, «las unidades de análisis representan los segmentos del contenido que son caracterizados e individualizados para posteriormente categorizarlos, relacionarlos y establecer inferencias a partir de ellos» ${ }^{(18)}$. 2. La codificación progresiva de todo el cuerpo de contenidos: se identificaron y agruparon en el texto las unidades de análisis según cada categoría atendiendo a características de exhaustividad, pertinencia y objetividad, las que posteriormente se introdujeron en tablas descriptivas por cada profesional médico.

El análisis descriptivo involucró tres niveles: En el primer nivel individual, por cada médico, consistió en identificar lo que cada unidad de análisis expresaba, agrupándolo en categorías e incorporando las emergentes en el discurso. En el segundo nivel de análisis, mediante lecturas repetidas, cruzadas y de comparaciones constantes se buscaron los patrones convergentes y divergentes, tendencias entre los discursos. Finalmente, el análisis descriptivo interpretativo de tercer nivel, última etapa en el análisis de contenido, que consistió en convertir la última columna del análisis de segundo nivel en el insumo del texto en la elaboración del capítulo de los resultados del estudio, el cual se estructuró conforme a la pregunta de investigación, dando respuesta a los objetivos del estudio.

\section{ASPECTOS ÉTICOS}

El proyecto original fue revisado y aprobado por el Comité del Programa de Doctorado en Ciencias de la Educación, Comité Ético de la Universidad de Santiago. Respecto a las valoraciones éticas en la investigación, se cumplieron los siguientes parámetros: firma de consentimiento informado, confidencialidad y privacidad, derecho a la no participación, y acceso a los resultados de la investigación.

\section{RESULTADOS}

Los hallazgos fueron agrupados en dos ejes temáticos: 1. Debilidades de la formación y 2. Percepción de las necesidades de formación para abordar la praxis médica en contextos multiculturales de salud pública.

El análisis del campo de trabajo médico en interacción con pacientes inmigrantes aporta elementos contextuales de los desafíos de esta práctica. En la Figura 1 se describe el impacto en salud pública de la condición de vulnerabilidad del inmigrante, como determinante de su salud y los desafíos a la atención médica. Destaca la percepción de cambio significado por las tensiones de una población crecientemente diversa, principalmente de rostro latinoamericano descrito en la Tabla 3.

Los médicos reconocen al paciente inmigrante, aunque no se consulta, ni registra la procedencia en las estadísticas ni en la ficha clínica. Esta situación no sólo lleva a problemas de registro estadístico, sino también contribuye a originar múltiples miradas sobre esta población, cargadas de subjetividad y prejuicios, según se expresa:

«hay una sensación de mayor consulta porque están constantemente aquí, día tras día» (Entrevista 5, varón, servicio de salud en Santiago).

Sin embargo, los participantes distinguen a los inmigrantes según grupos que caracterizan homogéneos entre sí, según estereotipos que predisponen al profesional a prejuicios en la RMP (Tabla 4).

\section{DEBILIDADES DE LA FORMACIÓN}

Se percibe una importante falta de formación para dar una atención apropiada a pacientes inmigrantes:

"Chile hace mucho tiempo que empezó a recibir a mucha gente de otros países y seguimos con la misma formación, entonces no estamos preparados para enfrentar a este tipo de pacientes» (Entrevista 10, femenino, servicio de salud de Independencia)

"Como te digo, te mentiría si te dijera que nos dieron alguna herramienta como para enfrentarnos a la población extranjera acá, no» (Entrevista 13, femenino, servicio de salud de Conchalí)

Al respecto, destacan la escasa formación en ámbitos de epidemiología, sistema sanitario y salud pública de los países de procedencia inmigrante y de América Latina: 


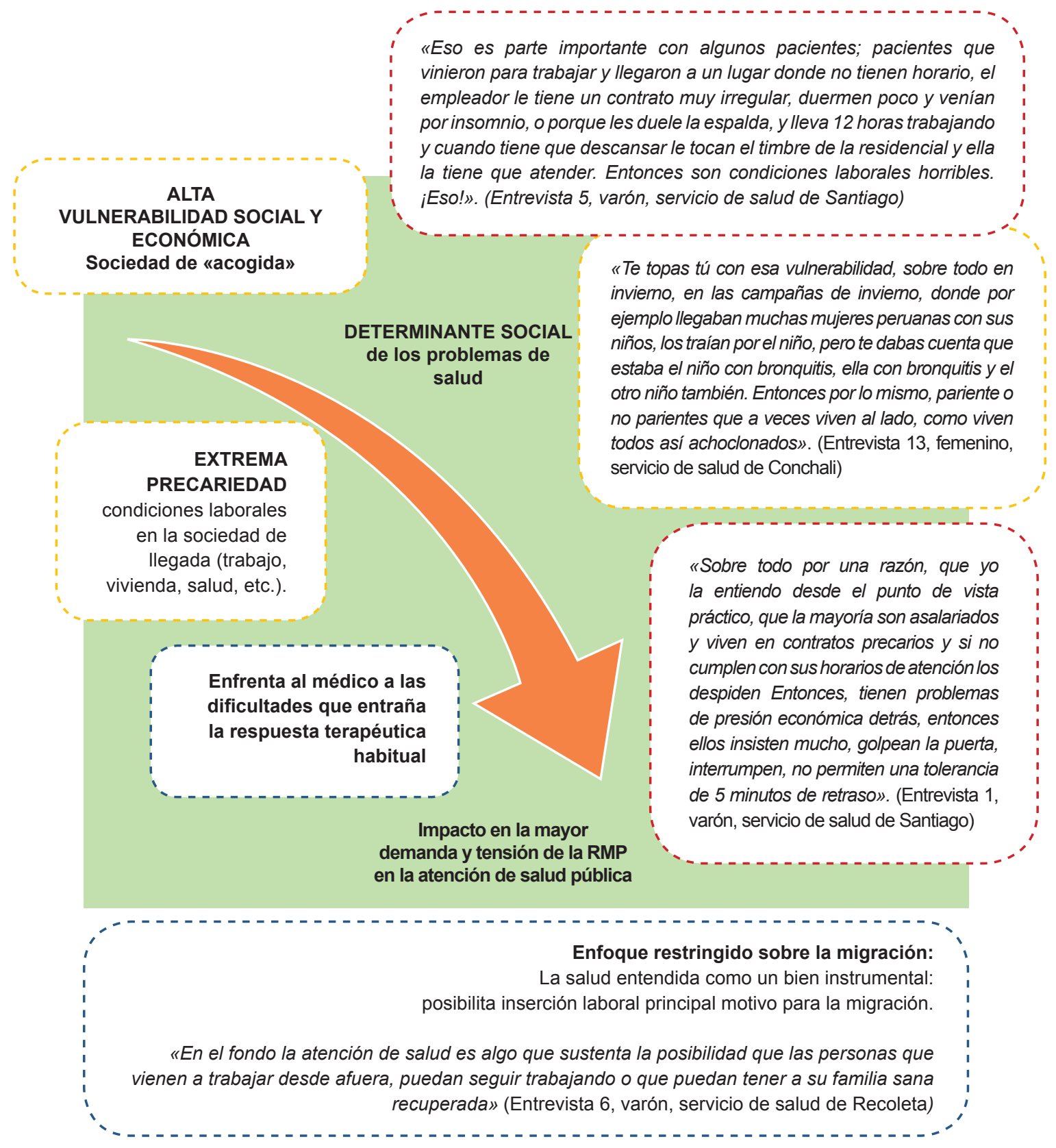

Figura 1. Impacto en salud pública por la condición de vulnerabilidad del inmigrante

«Porque uno no conoce mucho la epidemiología de todos los otros países que lo rodean a uno». (Entrevista 8, femenino, servicio de salud de Independencia)

«Pero sí, es importante que probablemente pueden tener otras enfermedades que tal vez nosotros no manejamos y de las cuales todavía no nos hacemos cargo». (Entrevista 7, femenino, servicio de salud de Independencia)

Consideran una debilidad en la formación actual el conocimiento centrado en la población general o chilena, la cual es homogénea culturalmente:
«En general el pregrado está orientado básicamente a la población chilena (...), no se nos enseña cómo abordar al paciente extranjero». (Entrevista 10, femenino, servicio de salud en Independencia).

Expresan que el modelo de formación médica con orientación al énfasis biomédico y acento en la especialización revela dificultades para la interculturalidad y la atención integral:

"Tu estás en tercero o cuarto y ya te están preguntando que especialidad quieres». (Entrevista 13, femenino, servicio de salud de Conchalí) 
Tabla 3. Percepción de los médicos acerca del contexto de relación médico-paciente inmigrante

\begin{tabular}{|c|c|}
\hline Percepción general & Cita textual \\
\hline $\begin{array}{l}\text { SOBREDEMANDANTE del servicio } \\
\text { de salud público. Incremento en: } \\
\text { - La demanda de atención médica } \\
\text { - Porcentaje de población y número de } \\
\text { atenciones otorgadas. } \\
\text { - La presión asistencial }\end{array}$ & $\begin{array}{l}\text { "¿Aquí? ¡Un incremento terrible! A veces un tercio de la atención es inmigrante. Hoy día } \\
\text { tengo aquí una lista aquí tremenda de } 20 \text { pacientes, de los cuales } 12 \text { son extranjeros. A veces } \\
\text { la atención es solamente de inmigrantes». (Entrevista 2, varón, servicio de salud de Santiago) } \\
\text { "Probablemente sea casi la mitad de la población que uno atiende, o sea al menos en el } \\
\text { número de atenciones diarias, probablemente lo sea». (Entrevista 12, varón, servicio de } \\
\text { salud de Santiago) }\end{array}$ \\
\hline $\begin{array}{l}\text { POLICONSULTANTE } \\
\text { No se llega a percibir la alta tasa de } \\
\text { inmigrante policonsultante como un } \\
\text { síntoma de que algo no funciona en la } \\
\text { atención }\end{array}$ & $\begin{array}{l}\text { "Además son policonsultantes, la mamá con hijo, o sea, uno ve que viene dos o tres } \\
\text { veces en una misma semana. No sabemos si vuelven porque no saben cómo manejar la } \\
\text { fiebre en la casa o no saben cómo manejar un resfrío en la casa. No sabemos si es que } \\
\text { vuelven porque no entendieron las explicaciones del colega o no quedaron conformes». } \\
\text { (Entrevista 4, femenino, servicio de salud de Santiago) } \\
\\
\text { "Sí!, muchos, bueno, acá en el Servicio de Urgencias en los últimos años, hay yo diría, } \\
\text { que una cantidad alta de inmigrante. Son poli consultantes, muy demandantes. No, uno } \\
\text { les explica, les tiene que volver a explicar, y le vuelven a consultar a uno, lo mismo que } \\
\text { uno le ha dicho. Son muy repetitivos en las consultas». (Entrevista 9. femenino, servicio } \\
\text { de salud de Independencia) }\end{array}$ \\
\hline $\begin{array}{l}\text { ATENTO A RECLAMAR SUS } \\
\text { DERECHOS }\end{array}$ & $\begin{array}{l}\text { "Si él (inmigrante) ve una diferencia, inmediatamente reclaman, y se hace respetar». } \\
\text { (Entrevista 2, varón, servicio de salud de Santiago) }\end{array}$ \\
\hline $\begin{array}{l}\text { CARACTERIZACIÓN NEGATIVA } \\
\text { DEL PACIENTE EXTRANJERO: } \\
\text { Demandante-Policonsultante } \\
\text { Exigente-Desconforme con la } \\
\text { atención. } \\
\text { Idea asociada: Mal paciente, no } \\
\text { responde como se espera, su } \\
\text { atención genera estrés al médico }\end{array}$ & $\begin{array}{l}\text { "Son bien demandantes en general, entonces es igual un paciente que es desgastante. } \\
\text { En general, son pacientes que quieren que todo se les solucione, que todo se le dé, } \\
\text { entonces no están dispuestos a invertir en algún examen que hay que hacer, o si de } \\
\text { repente hay que comprar algún medicamento, no!. Todo en general lo quieren, ese es el } \\
\text { tema y en eso se diferencia de la población chilena». (Entrevista 10, femenino, servicio } \\
\text { de salud de Independencia) }\end{array}$ \\
\hline $\begin{array}{l}\text { CONSTRUCCIÓN DE OTREDAD } \\
\text { Inferior del paciente inmigrante en } \\
\text { relación a la población chilena. } \\
\text { - Presencia de jerarquías asimétricas } \\
\text { en el discurso: lo nuestro, de aquí- } \\
\text { ellos; los chilenos-extranjeros (de otro } \\
\text { sitio); de afuera (intruso, no invitado) } \\
\text { - Conflicto en el campo clínico: el } \\
\text { medico se autoinstituye con los } \\
\text { nuestros y siente que los desplaza por } \\
\text { la irrupción inmigrante (los otros) } \\
\text { - Procede de una sociedad más } \\
\text { atrasada en lo civilizatorio (educación) } \\
\text { - Estratificación social y cultural } \\
\text { inferior }\end{array}$ & $\begin{array}{l}\text { «Es tanta la inmigración que dentro de nuestro grupo, muchas veces vemos que nuestras } \\
\text { pacientes a veces quedan rezagadas en la atención». (Entrevista 3, varón, servicio de } \\
\text { salud de Santiago) } \\
\text { "Es más pobre y menos educado que el pobre chileno»... (Entrevista 11, varón, servicio } \\
\text { de salud de Independencia) } \\
\text { «Probablemente el nivel cultural es mucho más bajo que nuestro consultante medio». } \\
\text { (Entrevista 1, varón, servicio de salud de Santiago) }\end{array}$ \\
\hline
\end{tabular}

Por su parte, la relevancia de la formación hospitalaria la torna más atractiva por su complejidad biomédica, generando una serie de expectativas sociales que fuerzan a privilegiar en el estudiante la orientación hacia la especialidad, más que a la atención primaria, incluso bajo criterios de rentabilidad:

"Yo creo que no hay interés en algo que no sea rentable. Pienso que la rentabilidad es el valor de ahora». (Entrevista 9, femenino, servicio de salud de Independencia).

"La formación de nosotros los médicos, no tiene tanta orientación a la APS. Hemos recibido un montón de formación hospitalaria y después llegas acá y no aplicas, entonces vas aprendiendo en el camino». (Entrevista 12, varón, servicio de salud de Santiago)
Destacan la formación teórica con escasas herramientas en habilidades de comunicación efectiva para enfrentar la complejidad de la práctica médica cotidiana con inmigrantes.

\section{PERCEPCIÓN DE LAS NECESIDADES DE FORMACIÓN}

Los médicos expresan con fuerza la necesidad de recibir formación para la atención pertinente a pacientes inmigrantes y pacientes con necesidades diversas (sordedad, género, diversidad sexual), de importancia relevante, porque el desconocimiento de estos aspectos se considera como un riesgo para la atención. 
Tabla 4. Percepción acerca de la procedencia de los pacientes inmigrantes en la relación médico-paciente inmigrante

\begin{tabular}{|c|c|}
\hline Procedencia & Cita textual \\
\hline & $\begin{array}{l}\text { "La gente de raza negra, son más altos y uno se da cuenta inmediatamente». (Entrevista 6, varón, } \\
\text { servicio de salud de Recoleta) }\end{array}$ \\
\hline Pacientes haitiano o dominicano & $\begin{array}{l}\text { "Ahora lo que sí, respecto de los pacientes que sean de República Dominicana o Haití, que son } \\
\text { negros o de color ¿no sé cómo se dice: negro o de color?, es que todos somos de color. De } \\
\text { partida ahí uno no sabe decir si es un paciente negrito o qué. Lo que sí es un paciente diferente, } \\
\text { pero con ellos, la verdad que en general, lo que yo veo del personal o los médicos es que les } \\
\text { encanta atenderlo. Siempre son como llamativos porque como que da una cierta simpatía; los } \\
\text { niñitos son muy lindos, las niñitas también, con sus peinados especiales, no vienen tampoco con } \\
\text { una actitud desafiante, ni nada; son bien como afables». (Entrevista 7, femenino, servicio de salud } \\
\text { de Independencia) }\end{array}$ \\
\hline Paciente dominicano & $\begin{array}{l}\text { «Después los de República Dominicana, ahí sí no he visto esta diferencia que hay con los peruanos, } \\
\text { como que siento que entienden más». (Entrevista 7, femenino, servicio de salud de Independencia) }\end{array}$ \\
\hline \multirow[t]{2}{*}{ Paciente peruano } & $\begin{array}{l}\text { «Pero es evidente que para uno es distinto saber que el paciente que viene es peruano o es } \\
\text { chileno. Porque si es peruano, uno sabe que la consulta va a ser más larga, va a costar más y } \\
\text { lo más probable que todo lo que tú hayas invertido en tiempo, en dedicación, no sirva de nada». } \\
\text { (Entrevista 9, femenino, servicio de salud de Independencia) }\end{array}$ \\
\hline & $\begin{array}{l}\text { "Los rasgos de los peruanos en general son más incaicos, tienen los ojos más rasgados, caras más } \\
\text { redondas, el pelo también es medio distinto, el color de la piel, generalmente son más morenos». } \\
\text { (Entrevista 6, varón, servicio de salud de Recoleta) }\end{array}$ \\
\hline Paciente ecuatoriano & $\begin{array}{l}\text { "Los ecuatorianos sobre todo, porque vienen de la sierra, del interior, y tienen un muy bajo nivel } \\
\text { cultural. Los que son más avispados, llamémoslo así, y más preparados, son indudablemente los } \\
\text { colombiano». (Entrevista 1, varón, servicio de salud de Santiago) }\end{array}$ \\
\hline Paciente boliviana & $\begin{array}{l}\text { "Las pacientes ecuatorianas si uno logra establecer una buena relación, la paciente retribuye, pero } \\
\text { la boliviana es como mucho más recelosa de la información que entrega». (Entrevista } 3 \text {, varón, } \\
\text { servicio de salud de Santiago) }\end{array}$ \\
\hline
\end{tabular}

«En ética se tiene que tocar el tema de atención, de cómo y cuan complejo puede ser, las herramientas que uno tiene y así de un paciente de una etnia, como un paciente de una elección sexual distinta a la que uno espera o un paciente que sea de un país extranjero, que tenga una connotación previa. Esas prestaciones tienen que ser de igual calidad para todos ellos, y para todos ocupar herramientas distintas». (Entrevista 1, varón, servicio de salud de Santiago).

En resumen, los médicos enfrentados a los desafíos sanitarios de la creciente migración sugieren que la formación de pregrado incorpore los siguientes aspectos:

- El fenómeno de la migración internacional en el contexto histórico de Chile y Latinoamérica:

"Si nosotros supiéramos que están llegando todos los años tanto inmigrante al país». (Entrevista 4, femenino, servicio de salud Santiago)

\section{- Perspectiva global de la salud pública desde América Latina:}

«Pero tenemos que conocer a la gente, yo creo que tenemos que saber de dónde vienen, qué enfermedades podrían eventualmente tener, como para que nosotros nos preparemos». (Entrevista 7 , femenino, servicio de salud de Independencia)

"Tener un poquito más de idea de epidemiología peruana, asi como saber qué prevalencias tienen ellos, eso nos ayudaría un montón. Por darte un ejemplo, o sea (peruana), que es la mayor masa que llega acá del extranjero. Sí, sería súperbueno saber qué epidemiología tienen ellos, qué tasas manejan, hasta el solo hecho de saber qué vacunas tienen, o sea, que son distintos a los programas de educación que tenemos nosotros». (Entrevista 13, femenino, servicio de salud de Conchalí)
- Conocimiento acerca las enfermedades infecciosas que «no existen en Chile», o no están «acostumbrados a ver»:

"Conocer más las enfermedades tropicales, hay gente que ha llegado con malaria. La malaria uno no la ve acá, uno no la trata o algunas otras infecciones, parasitosis que no tenemos acá en Chile. Eso quizás tiene que ver más con el lugar de origen ambiental, el ecosistema de donde viene el usuario. Ahora, por ejemplo, los alimentos también son importantes, porque por ejemplo hay zonas donde hay más parásitos intestinales, comen el pescado más crudo o hay diarreas. La epidemiología es muy importante, la epidemiología de las enfermedades, cómo sea en los países de origen». (Entrevista 6, varón, servicio de salud de Recoleta)

- La migración como un derecho humano y temáticas como la xenofobia, la discriminación, coherencia con el currículo oculto de la formación.

\section{DISCUSIÓN}

De acuerdo a los médicos entrevistados, el aumento de la población inmigrante es un nuevo desafío a la atención médica y a la formación de los futuros profesionales de la salud. Destacan la falta de formación que les permita situarse adecuadamente al nuevo contexto y brindar una atención pertinente a las necesidades de la creciente población extranjera, cada vez más heterogénea en su composición. Se revela la importancia de la barrera idiomática en la relación, al igual que en los estudios sobre el tema y la necesidad de desarrollo de la capacidad de 
comunicación intercultural en la formación orientada a problemas reales del proceso de RMP.

Las necesidades percibidas de formación por los participantes médicos en la atención del inmigrante se refieren a tres grandes áreas: 1. El fenómeno de la migración internacional y la salud pública, dinámica latinoamericana. 2. Epidemiologia y enfermedades en países de origen de los inmigrantes. 3. Perspectiva de derechos humanos de la migración internacional.

El estudio acerca de los perfiles de egreso de carreras de medicina de Chile, pone en evidencia que no están considerando a la política pública, ni los objetivos sanitarios como un eje movilizador de su formación, lo cual genera una gran duda respecto a la articulación de la educación médica con las necesidades de salud del país. Se evidencia en la formación médica, la centralidad de la enseñanza en el hospital, el marcado acento en la especialización, todo lo cual implica obstáculos para incorporar las orientaciones de la Organización Panamericana de la Salud en la formación médica ${ }^{(19)}$. Este paradigma de la formación no considera la creciente diversidad inmigrante, como tampoco la realidad de personas con necesidades diferentes, que no aborda universidad alguna y que el médico debe enfrentar en su quehacer habitual.

De acuerdo a los hallazgos, la compleja dinámica de la praxis intercultural en contextos multiculturales, abre perspectivas nuevas a la Educación Médica desde tres ámbitos: 1) El marco de los derechos humanos: la salud es un derecho irrenunciable pero que requiere de un diálogo intercultural acerca de la dignidad humana ${ }^{(20)}$. El Ministerio de Salud de Chile, plantea el enfoque intercultural y de derechos de la salud pública, reconociendo como sujetos de derechos a los usuarios inmigrantes ${ }^{(21)}$. 2) La misión social de integración de los inmigrantes a la sociedad de acogida, que puede reducir el surgimiento de actitudes xenofóbica, dado que el inmigrante enfermo puede ser visto por la población receptora como una amenaza y potencial fuente de contagio de enfermedades ${ }^{(22)}$. 3) El enfoque intercultural para la paz en contextos multiculturales de salud pública y el desarrollo de la medicina intercultural ${ }^{(23)}$

El presente estudio tiene limitaciones propias de la investigación cualitativa, el tamaño de la muestra no posibilita generalizar los resultados obtenidos. Sin embargo, esta composición permitió ahondar en la profundidad del conocimiento del objeto de estudio. Otra de las debilidades es lo limitado del tiempo que impide aprehender la evolución y complejidad del proceso dinámico de RMP inmigrante y la adaptación intercultural del médico que evoluciona a diario en la praxis multicultural de salud pública, lo cual va generando nuevas demandas a la formación médica en un contexto dinámico de la política pública. En cuanto a las fortalezas del estudio, pretende ser un aporte en la discusión acerca del desafío que enfrenta la formación médica ante la creciente población migrante, y la necesidad de problematizar la RMP para una educación intercultural.

Se puede concluir que el debate acerca de la interculturalidad en la formación médica levanta dimensiones críticas que se articulan con la política pública del país y los nuevos desafíos que la migración internacional plantea a la salud pública.

Conflictos de interés: la autora declara no tener conflictos de interés.

Fuentes de financiamiento: autofinanciada

\section{REFERENCIAS BIBLIOGRÁFICAS}

1. Ministerio de Desarrollo Social de Chile. Encuesta de Caracterización Socioeconómica Nacional (CASEN). Inmigrantes en Chile: Perfil socioeconómico por macrozona de residencia. 2015. [citado 2018 enero 22]. Disponible en http://observatorio.ministeriodesarrollosocial.gob.cl

2. Departamento de Extranjería y Migración. Ministerio del Interior y Seguridad Pública. DEM. Informe La migración en Chile: breve reporte y caracterización Julio/agosto 2016. [citado 2018 enero 22] Disponible en http://www.extranjeria. gob.cl/media/2016/08/informe_julio_ agosto_2016.pdf

3. Cabieses B, Bernales M, McIntyre A. M. La migración internacional como determinante social de la salud en Chile: evidencia y propuestas para políticas públicas. Universidad del Desarrollo, Fondecyt; 2017.
4. Tijoux M. E. Racismo en Chile. La piel como marca de la inmigración. Editorial Universitaria: Santiago Chile; 2016.

5. Achotegui J, Jayme M. El reto de trabajar la relación terapéutica extendida y la transferencia negativa con inmigrantes, minorías y excluidos sociales The challenge of working on the extended therapeutic relation and the negative transfer with. Clínica. 2016;7(2):105-112.

6. Thomas F, Gideon J. Migration, Health and Inequality. London, Zed Books: 2014.

7. Cortez Ana. Migración internacional: un desafío para las políticas públicas de Chile. En: Niñas y niños migrantes. Políticas públicas, integración e interculturalidad. Santiago, Chile: Ed. Colectivo Sin Fronteras; 2008.

8. Rodríguez-Álvarez E, González-Rábago Y, Bacigalupe A, Martín U, Lanborena-Elordui $N$. Inmigración y salud: desigualdades entre la población autóctona e inmigrante en el País Vasco. Gac Sanitaria. 2014;28(4):274-280.

9. Liberona-Concha NP. De la alterisación a la discriminación en un sistema público de salud en crisis: conflictos interétnicos a propósito de la inmigración sudamericana en Chile. Rev Ciencias Sociales (CI). 2012; 28,1-20.

10. Cabieses B, Bernales M, Obach A, Pedrero V. Vulnerabilidad social y su efecto en salud en Chile. Desde la comprensión del fenómeno hacia la implementación de soluciones. Universidad del Desarrollo; 2016.

11. Alarcón AM, Vidal A, Neira J. Salud intercultural: elementos para la construcción de sus bases conceptuales. Rev Med Chil. 2003;131(9):1061-5.

12. Quilaqueo D, Torres H. Multiculturalidad e interculturalidad: desafíos episte- 
mológicos de la escolarización desarrollada en contextos indígenas. Alpha(Osorno) 2013;(37),285-300.

13. Rodríguez-Martin Beatriz. Desafíos y oportunidades de las situaciones de contacto cultural en el ámbito sanitario. Index Enferm. 2015;24(4):227-231.

14. Zemelman H. Voluntad de conocer. El sujeto y su pensamiento en el paradigma crítico. Barcelona, España: Ed. Anthropos; 2005

15. Vasilachis I. Estrategias de Investigación Cualitativa. Barcelona. España: Gedisa Editorial; 2006.

16. Margarit D, Bijit K. Barrios y población inmigrantes: el caso de la comuna de Santiago. Revista INVI. 2014; 29 (81): 19-77.

17. Taylor SJ, Bogdan R. Introducción a los métodos cualitativos de investigación: La búsqueda de significados. Editorial Paidós Básica. 1987.

18. Cáceres P. Análisis cualitativo de contenido: una alternativa metodológica alcanzable. Psicoperspectivas. Individuo y Sociedad. 2008;2(1):53-82.

19. Parada M, Romero MI, Moraga F. Perfiles de egreso de las carreras de Medicina en Chile. Rev Med Chile. 2015; 143(4): 512-519.

20. Sousa Santos B. Descolonizar el saber, reinventar el poder. Santiago Chile: LOM Ediciones; 2013.

21. Ministerio de Salud de Chile. Estrategia Nacional de salud. Para el cumplimiento de los Objetivos Sanitarios de la década 20112010.
22. Zepeda-Ortega A, Malena-Monteverde L. La inmigración en Chile: Una mirada desde los Determinantes Sociales de la Salud. [online]. 2014 [citado 2018 enero 20]. Disponible en: http://actacientifica. servicioit.cl/biblioteca/gt/GT9/GT9_ ZepedaAMalenaL.pdf.

23. EscrigM. Lasalud dela población inmigrante en España: marginación, exclusión, desafios y realidades. Revista de Trabajo Social y Salud. 2008; 59: 1-18.

\section{Correspondencia: Ruth Urrutia Arroyo.}

Dirección: Universidad Católica Silva Henriquez. Casa Central. General Jofre 462., Santiago. Teléfono: 954441738.

Correo electrónico:ruth.urrutia@gmail.com

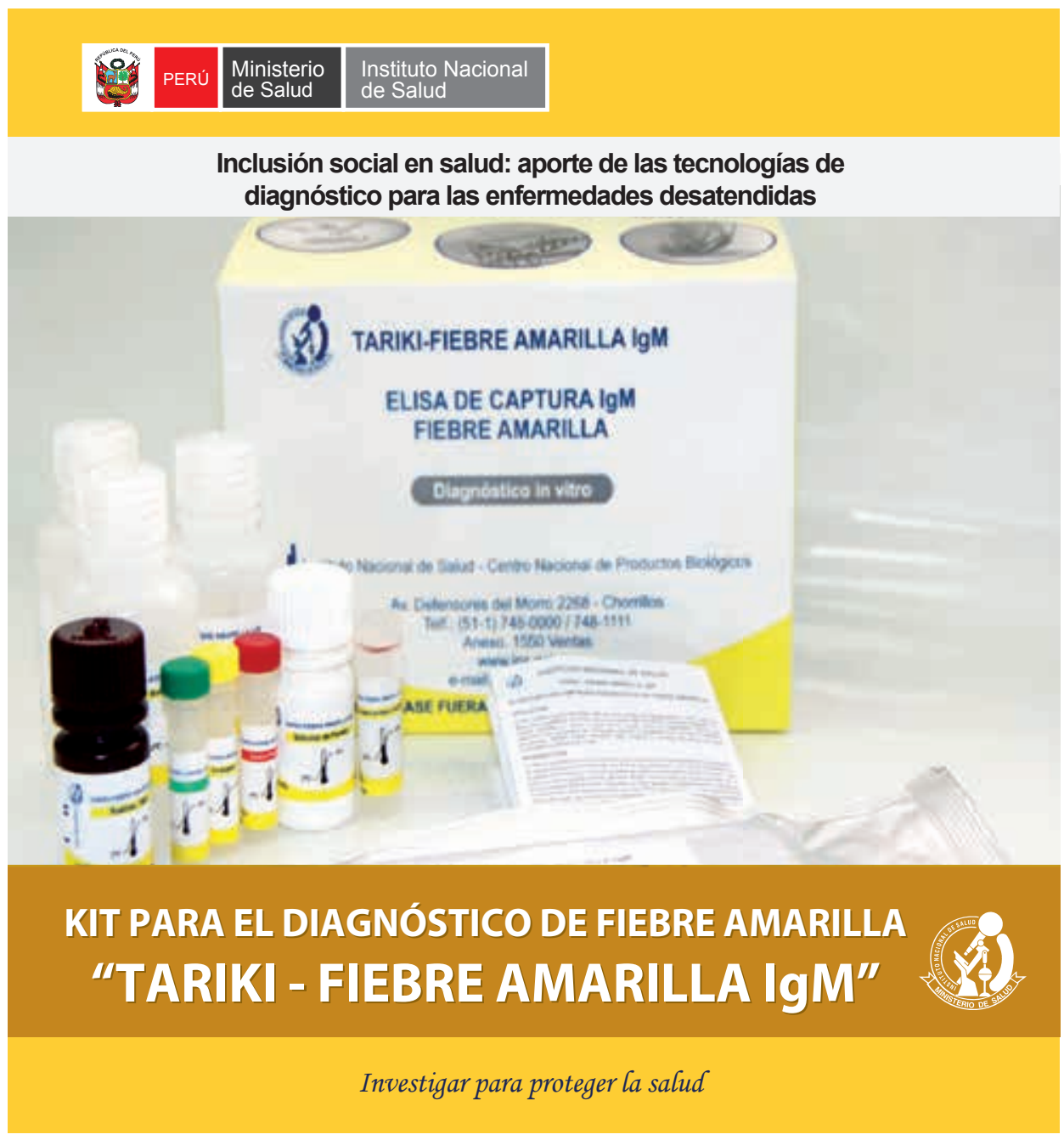

